\title{
A Severe Case of Anaplastic Large Cell Lymphoma in a Previously Healthy Woman: Diagnostic and Therapeutic Challenges
}

\author{
Petya Angelova ${ }^{1}$, Robert Brock ${ }^{1}$, Alexandra-Teodora Negoescu ${ }^{1}$, \\ Lara-Marie Schmitt' ${ }^{2}$ Julia Meißner², Sascha Dietrich², \\ Carsten Müller-Tidow ${ }^{2}$, Jan Philipp Novotny ${ }^{2}$ \\ ${ }^{1}$ Heidelberg Medical Faculty, Heidelberg University, Heidelberg, Germany; \\ ${ }^{2}$ Department of Internal Medicine V, University Hospital Heidelberg, Heidelberg, \\ Germany
}

Received May 9, 2020; Accepted November 2, 2020.

Key words: Lymphoma - Anaplastic - Anaplastic large cell lymphoma Hemophagocytic lymphohistiocytosis

Abstract: Anaplastic large cell lymphomas are an aggressive subtype of peripheral T-cell lymphomas that can manifest with a variety of symptoms. Our case highlights the importance of prompt tissue sampling, especially if an associated hemophagocytic lymphohistiocytosis is detected and no clinical improvement is observed upon glucocorticoid treatment.

Mailing Address: Jan Philipp Novotny, MD., PhD., Department of Internal Medicine V, University Hospital Heidelberg, Im Neuenheimer Feld 410, 69120 Heidelberg, Germany; Phone: 062215 60;

e-mail: JanPhilipp.Novotny@med.uni-heidelberg.de 


\section{Introduction}

Anaplastic large cell lymphomas (ALCL) are pathologically distinct lymphoid neoplasms with clinically aggressive behaviour. ALCL show an incidence of approximately 0.25 per 100,000 person-years and account for approximately $14 \%$ of all diagnosed NK/T-cell lymphomas (Morton et al., 2006). The anaplastic lymphoma kinase $(A L K)$ is aberrantly expressed in approximately 50 to $80 \%$ of all patients with $A L C L$ and correlates with chromosomal rearrangement, e.g. $\mathrm{t}(2 ; 5)(\mathrm{p} 23 ; \mathrm{q} 35)$ - resulting in an ALK-NPM1 fusion. It allows the subdivision into ALK-positive and ALK-negative ALCL, which are listed as distinct entities in the current World Health Organization classification (Swerdlow et al., 2008). In a retrospective analysis, the median age of ALK-positive ALCL was 34 years, whereas the median age of ALK-negative ALCL was 58 (Savage et al., 2008). For both subsets, patients are predominantly male and present with stage III to IV disease, which often involves multiple extranodal sites including skin, soft tissue, bone, lung, intestinal tract, liver, spleen and bone marrow (Falini et al., 1999; Savage et al., 2008; Sibon et al., 2012). B-symptoms and other paraneoplastic phenomena like migratory rash, pruritus, arthralgias, lymph node, and skeletal pain are commonly observed (Shustov and Soma, 2019). Initial laboratory analyses sometimes reveal nonspecific abnormalities, such as lactate dehydrogenase (LDH) elevation, anemia and thrombocytopenia (Savage et al., 2008). Usually the diagnosis of $A L C L$ is suggested by the morphology and confirmed by positive immunohistochemistry for CD30 and eventually ALK (Shustov and Soma, 2019). Early recognition and diagnosis followed by appropriate therapy are critical cornerstones of care. Treatment with Brentuximab vedotin, Cyclophosphamide, Doxorubicin, and Prednisone (A+CHP) can entail a similar rate of adverse effects like febrile neutropenia or peripheral neuropathy, but was shown to be associated with a significantly improved progression free survival when compared to standard CHOP (Vincristine, Doxorubicin, Cyclophosphamide, Prednisone) therapy (Horwitz et al., 2019).

A rare and severe complication of ALCL is hemophagocytic lymphohistiocytosis $(\mathrm{HLH})$. This cytokine-driven hyperinflammatory syndrome can either manifest primarily due to a genetic defect or as a secondary concomitant phenomenon of hematological malignancies, infections, autoimmune processes or drug intake (Vick et al., 2017). HLH is diagnosed in about 23\% of all patients with peripheral T-cell lymphomas and is associated with a significantly worse prognosis (Xie et al., 2013). The median overall survival in these cases is less than 1 year and HLH can present with various symptoms. Thus, the clinical diagnosis requires the fulfilment of 5 out of 8 criteria (Vick et al., 2017). Laboratory findings showing cytopenia, hypertriglyceridemia, hyperferritinemia, and elevated soluble IL-2 receptor (sCD25) should heighten the suspicion of HLH. 


\section{Case report}

A previously healthy Caucasian female in her early fifties was referred by her general practitioner and presented with a one-week history of fever, headache, myalgia, as well as a three-week history of a slightly enlarged and tender right inguinal lymph node. The patient declined any travel history, night-sweat or loss of weight, but stated to suffer from fever (up to $40^{\circ} \mathrm{C}$ ) and shivering during the last week. Upon physical examination, enlarged cervical, supraclavicular and inguinal lymph nodes were observed. Initial laboratory work-up showed slight leukocytopenia $\left(2.89 \times 10^{9} /\right.$, range $4-10 \times 10^{9} /$ I) with left shift (banded neutrophils 14\%, segmented neutrophils $40 \%$, lymphocytes $38 \%$, monocytes $1 \%$, metamyelocytes $1 \%$, myelocytes $2 \%$, atypical lymphocytes $4 \%)$, as well as thrombocytopenia $\left(82 \times 10^{9} /\right.$, range $\left.150-440 \times 10^{9} / \mathrm{I}\right)$ and increased lactate dehydrogenase $(\mathrm{LDH})$ of $793 \mathrm{U} / \mathrm{I}$ (range $<308 \mathrm{U} / \mathrm{I})$ ). Furthermore, we observed an increased C-reactive protein (CRP) concentration of $28.9 \mathrm{mg} / \mathrm{I}$ (range $<5 \mathrm{mg} / \mathrm{l})$, minimally elevated aspartate-aminotransferase (AST) and elevated lipase and pancreatic amylase, albeit without associated symptoms of pancreatitis. Empirical antibiotic therapy was started with amoxicillin/clavulanic acid and subsequently changed to ceftriaxone and clarithromycin due to persistent fever.

Based on these findings we initially suspected an infectious cause and abdominal sonography revealed hepatosplenomegaly, which was consistent with a possible Epstein-Barr-Virus (EBV) infection. Computed tomography of the neck, chest and abdomen additionally demonstrated several marginally or pathologically enlarged lymph nodes. However, analyses of anti-EBV IgG and IgM demonstrated only positivity for gamma immunoglobulin, indicating a past infection. Further workup did not reveal any signs of an active or past HIV, cytomegalovirus (CMV) or hepatitis B/C infection and blood cultures remained without microbial growth. We therefore performed a bone marrow aspiration, which showed maturation of all cell-lines. However, toxic granulations were observed within the granulopoietic system and slight dysplastic changes of erythropoiesis, but without the presence of ring sideroblasts or cytologically apparent neoplastic infiltration. In the course of the disease, the patient rapidly developed progressive hyponatremia, as well as markedly elevated liver enzymes (AST up to 2,486 U/I, range $<37 \mathrm{U} / \mathrm{I}$, ALT 1,551 U/I, range < $35 \mathrm{U} / \mathrm{I}$, and GGT 1,136 U/I, range < $40 \mathrm{U} / \mathrm{I})$. Concomitantly, we observed worsening of the international normalized ratio (INR), rise in LDH (up to $3,041 \mathrm{U} / \mathrm{I}$ ), soluble IL-2 (up to $34,864 \mathrm{U} / \mathrm{ml}$ ) and ferritin (up to 20,066 $\mu \mathrm{g} / \mathrm{I}$ ) - indicating an underlying hemophagocytic lymphohistiocytosis (HLH) with hepatic involvement. The most common secondary infectious causes of HLH were ruled out by extensive serological and microbiological testing. We started the patient on $100 \mathrm{mg} /$ day intravenous prednisone, after which liver transaminases decreased. However, the patients' overall condition did not improve and she suffered from constant fever, even with continuous application of novaminsulfon.

Concomitant pathological analyses of an inguinal lymph node, as well as bone marrow biopsy and liver biopsy then revealed a highly proliferating (Ki67: 90-95\%) 
neoplasm with medium-sized cytomorphology. Immunohistochemistry showed positivity for CD30, ALK-1, Perforin, EMA, CD3 and weak CD4, whilst no signal was observed for CD20, PAX-5, CD68. CD117, CD138, CD5, CD8 and AE1/3. Therefore, the diagnosis of a highly proliferating ALK-positive anaplastic large cell lymphoma (ALCL) was made. Furthermore, marked necroinflammatory and proliferative activity of the liver parenchyma was observed.

We immediately started a treatment with dose-adapted A+CHP (Brentuximab, Cyclophosphamide, Doxorubicin and Prednisone), after which we did not observe a tumour lysis syndrome according to the Cairo-Bishop criteria, but a decrease in renal function with a glomerular filtration rate of minimally $28.5 \mathrm{ml} / \mathrm{min} / 1.73 \mathrm{~m}^{2}$ according to CKD-EPI and hydropic decompensation that necessitated aggressive diuretic therapy, as well as supportive oxygen. Due to rising CRP and recurrent fever, antibiotic therapy was then escalated to piperacillin/tazobactam and subsequently to meropenem. Computed tomography of the chest revealed bipulmonary infiltrates. We transferred the patient to our intensive care due to progressive respiratory insufficiency and endotracheal intubation was necessary thereafter. The patients' condition worsened with concomitantly rising liver parameters, CRP, LDH and progressive renal insufficiency. Blood cultures showed growth of Enterococcus faecium, but no significant growth of pathological microbiota was observed after culture of upper respiratory tract material. Despite maximum catecholaminergic support and anti-infective treatment according to the obtained antibiogram, the patient died of (most likely) pneumogenic neutropenic septic shock with multi-organ failure only 8 days after diagnosis of the underlying ALCL.

\section{Discussion and Conclusion}

Hodgkin and non-Hodgkin lymphomas like ALCL can present diagnostic challenges for clinicians. Due to the rarity and broad differential diagnosis these malignancies can remain clinically unrecognized over a long period of time (Mosunjac et al., 2008). Since the clinical symptoms and initial laboratory analyses of patients with ALCL are nonspecific and vary considerably among individuals, immunohistochemical analysis of affected sites is required to confirm the diagnosis (Hapgood and Savage, 2015). Furthermore, a unique feature of our case was that $A L C L$ presented as an inguinal lymph node which was painful, in contrast to lymphomatous nodes, which usually appear to be non-tender (Bilodeau and Fessele, 1998).

The patient's history of prolonged fever and tender inguinal lymph node thus also pointed to an infectious disease as the trigger of her hemophagocytic lymphohistiocytosis. Furthermore, treatment with prednisone did not result in clinical improvement in our patient, which is in contrast to its observed beneficial effects in patients with lymphoid malignancies (Pufall, 2015).

In summary, ALCL should be suspected whenever a patient presents with fever, mild lymphadenopathy, hepatosplenomegaly and laboratory findings consistent with a hyperinflammatory syndrome. Our case report highlights the importance of timely 
collection of bioptic material for the early detection and correct identification of an underlying ALCL. Furthermore, maintaining a high degree of suspicion is decisive to identify a hematologic malignancy as the underlying cause for hemophagocytic lymphohistiocytosis because clinical symptoms can overlap.

\section{References}

Bilodeau, B. A., Fessele, K. L. (1998) Non-Hodgkin's lymphoma. Semin. Oncol. Nurs. 14, 273-283.

Falini, B., Pileri, S., Zinzani, P. L., Carbone, A., Zagonel, V., Wolf-Peeters, C., Verhoef, G., Menestrina, F., Todeschini, G., Paulli, M., Lazzarino, M., Giardini, R., Aiello, A., Foss, H. D., Araujo, I., Fizzotti, M., Pelicci, P. G., Flenghi, L., Martelli, M. F., Santucci, A. (1999) ALK+ lymphoma: Clinico-pathological findings and outcome. Blood 93, 2697-2706.

Hapgood, G., Savage, K. J. (2015) The biology and management of systemic anaplastic large cell lymphoma. Blood 126, 17-25.

Horwitz, S., O'Connor, O. A., Pro, B., Illidge, T., Fanale, M., Advani, R., Bartlett, N. L., Christensen, J. H., Morschhauser, F., Domingo-Domenech, E., Rossi, G., Kim, W. S., Feldman, T., Lennard, A., Belada, D., Illes, A., Tobinai, K., Tsukasaki, K., Yeh, S. P., Shustov, A., Huttmann, A., Savage, K. J., Yuen, S., lyer, S., Zinzani, P. L., Hua, Z., Little, M., Rao, S., Woolery, J., Manley, T., Trumper, L., Group, E.-S. (2019) Brentuximab vedotin with chemotherapy for CD30-positive peripheral T-cell lymphoma (ECHELON-2): A global, double-blind, randomised, phase 3 trial. Lancet 393, 229-240.

Morton, L. M., Wang, S. S., Devesa, S. S., Hartge, P., Weisenburger, D. D., Linet, M. S. (2006) Lymphoma incidence patterns by WHO subtype in the United States, 1992-2001. Blood 107, 265-276.

Mosunjac, M. B., Sundstrom, J. B., Mosunjac, M. I. (2008) Unusual presentation of anaplastic large cell lymphoma with clinical course mimicking fever of unknown origin and sepsis: autopsy study of five cases. Croat. Med. J. 49, 660-668.

Pufall, M. A. (2015) Glucocorticoids and cancer. Adv. Exp. Med. Biol. 872, 315-333.

Savage, K. J., Harris, N. L., Vose, J. M., Ullrich, F., Jaffe, E. S., Connors, J. M., Rimsza, L., Pileri, S. A., Chhanabhai, M., Gascoyne, R. D., Armitage, J. O., Weisenburger, D. D.; International Peripheral T-Cell Lymphoma Project (2008) ALK- anaplastic large-cell lymphoma is clinically and immunophenotypically different from both ALK+ ALCL and peripheral T-cell lymphoma, not otherwise specified: Report from the International Peripheral T-Cell Lymphoma Project. Blood 111, 5496-5504.

Shustov, A., Soma, L. (2019) Anaplastic large cell lymphoma: Contemporary concepts and optimal management. Cancer Treat. Res. 176, 127-144.

Sibon, D., Fournier, M., Briere, J., Lamant, L., Haioun, C., Coiffier, B., Bologna, S., Morel, P., Gabarre, J., Hermine, O., Sonet, A., Gisselbrecht, C., Delsol, G., Gaulard, P., Tilly, H. (2012) Long-term outcome of adults with systemic anaplastic large-cell lymphoma treated within the Groupe d'Etude des Lymphomes de l'Adulte trials. J. Clin. Oncol. 30, 3939-3946.

Swerdlow, S. H., Campo, E., Harris, N. L., Jaffe, E. S., Pileri, S. A., Stein, H. (2008) WHO Classification of Tumours of Haematopoietic and Lymphoid Tissues. IARC Press, Lyon.

Vick, E. J., Patel, K., Prouet, P., Martin, M. G. (2017) Proliferation through activation: Hemophagocytic lymphohistiocytosis in hematologic malignancy. Blood Adv. 1, 779-791.

Xie, W., Hu, K., Xu, F., Zhou, D., He, J., Shi, J., Luo, Y., Zhu, J., Zhang, J., Lin, M., Ye, X., Huang, H., Cai, Z. (2013) Clinical analysis and prognostic significance of lymphoma-associated hemophagocytosis in peripheral T cell lymphoma. Ann. Hematol. 92, 481-486. 